\title{
Morphology Control of Polymer Microspheres Containing Block Copolymers with Seed Polymerization
}

\author{
Hossein Taherzadeh, Shunsuke Sotowa, Kenji Ogino* \\ Graduate School of Bio-Applications and Systems Engineering, Tokyo University of Agriculture and Technology, \\ Koganei, Tokyo, Japan \\ Email: ${ }^{*}$ kogino@cc.tuat.ac.jp
}

Received 6 March 2015; accepted 29 March 2015; published 1 April 2015

Copyright (C) 2015 by authors and Scientific Research Publishing Inc.

This work is licensed under the Creative Commons Attribution International License (CC BY). http://creativecommons.org/licenses/by/4.0/

(c) (i) Open Access

\begin{abstract}
Microspheres based on binary polymer blend consisting of polystyrene (PSt), poly (methyl methacrylate) (PMMA), block copolymer comprising PSt and PMMA subunits, and ternary polymer blend consisting of PSt, PMMA, and block copolymer were fabricated by a solvent evaporation method, in which a polymer solution in dichloromethane was dispersed in water phase with the aid of a homogenizer to obtain an $0 / \mathrm{W}$ emulsion followed by solvent evaporation with agitation to solidify the polymer. In the case of ternary blend, the effect of block copolymer content on the morphology of resulting spheres was investigated. Ternary blends afforded the bi-compartmental morphologies, the intermediate morphology between Janus and core-shell, which was confirmed by TEM observation. Seed polymerization of St or MMA was also carried out utilizing the resulting microspheres as seed particles in order to control the shape, and the surface morphology of particles. The particles with snowman-like morphology were obtained by seed polymerization of St using PSt/PMMA binary blend microspheres as seed particles. Surface roughness was controlled by the polymerization of MMA in the block copolymer seed, and that of St in the ternary blend seed.
\end{abstract}

\section{Keywords}

Microsphere, Morphology, Polymer Blend, Block Copolymer, Seed Polymerization

\section{Introduction}

Micron-sized, monodispersed polymer particles are of great interest for a wide variety of applications, for ex-

${ }^{*}$ Corresponding author.

How to cite this paper: Taherzadeh, H., Sotowa, S. and Ogino, K. (2015) Morphology Control of Polymer Microspheres Containing Block Copolymers with Seed Polymerization. Open Journal of Organic Polymer Materials, 5, 43-50.

http://dx.doi.org/10.4236/ojopm.2015.52005 
ample, in the biomedical and environmental fields and in separation sciences [1]-[4]. In past decades, several polymerization techniques have been utilized to prepare polymeric particles with different size, such as emulsion polymerization, soap-free emulsion polymerization, suspension polymerization, dispersion polymerization and seeded emulsion polymerization. Among these, seeded emulsion polymerization is a good method for the preparation of particles with unique morphologies, such as multilayered core-shell [5]-[12], hemispheres [13] [14], and hollow structures [15] [16]. Okubo et al. reported seeded dispersion polymerization affording microspheres with specific shapes, such as snowman/confetti-like [17], hamburger-like [18], and disk-like [19].

It is also expected that particles based on block copolymers and polymer blends exhibit specific morphologies resulting from phase separations. In order to fabricate nanoparticles with inner micro-phase separated structure, Yabu et al. reported self-organized precipitation method [20] [21] in which a good solvent was evaporated from a block copolymer solution containing poor and good solvents. As for micron sized particles, the other type of solvent evaporation method has been used. In this method, a polymer solution is dispersed in an aqueous medium, and then the solvent is evaporated to solidify the polymer [22]-[24]. Phase-separation during the solvent evaporation afforded core-shell, and inverted core-shell, microdomain, hemisphere morphologies [22], and nonspherical shape of polystyrene/poly(methyl methacrylate) composite [23] [24].

In order to afford novel functionality to polymeric microspheres, it is important to control surface and inner morphology as well as the chemical structure, particle size and its distribution. In general block copolymers show nanometer sized microphase-separated structure, whereas simple polymer blends afford micrometer sized macrophase-separated structure. The addition of A-B block copolymer to the polymer blend consisting of homopolymers A and B decreases the domain size. Since phase separation in microspheres occurs in a limited space with large interface, it's possible to generate specific surface and inner morphology compared with bulk samples. Thus, it's important to understand the relationship between the fabrication conditions of microspheres and resulting morphology. In this paper, surface and inner morphologies were investigated for the microspheres based on the blends consisting of polystyrene (PSt), poly(methyl methacrylate) (PMMA) and PS- $b$-PMMA, and seed polymerizations of St and MMA were also conducted utilizing blend particles as the seed particles.

\section{Experimental}

\subsection{Materials}

Styrene (St), methyl methacrylate (MMA) (Wako Chemical, Japan), 1,1-diphenyletylene (DPE) (TCI, Japan) were purified by distillation under vacuum and stored in refrigerator before used. Tetrahydrofuran (Wako Chemical) was used as freshly distilled over sodium and benzophenone. sec-Butyl lithium (sec-BuLi) (1M in hexane) (Kanto Chemical, Japan), poly(vinyl alcohol) (PVA) (Kuraray, PVA224) as a stabilizer, 2,2'-azobis(isobutylronitrile) (AIBN) (TCI, Japan) as an initiator for radical seeded polymerization, sodium dodecyl sulfate (SDS) (TCI, Japan) as an emulsifier were used as received.

\subsection{Anionic Polymerization of Styrene}

After the evacuation followed with backfilling of nitrogen, $100-\mathrm{mL}$ two-necked flask containing a magnetic stirrer bar was charged with dry THF $(50 \mathrm{~mL})$ under nitrogen atmosphere with a gas-tight syringe. After the temperature was down to $-78^{\circ} \mathrm{C}$ with a dry ice/acetone bath, $0.96 \mathrm{~mL}(0.96 \mathrm{mmol})$ of $\mathrm{sec}-\mathrm{BuLi}$, and $10.1 \mathrm{~mL}$ of St $(0.096 \mathrm{~mol})$ were successively injected to the flask with gas-tight syringes. After $1 \mathrm{~h}$, the reaction mixture was precipitated into methanol. The product was filtrated, washed with methanol and dried in vacuo. Yield: $9.28 \mathrm{~g}$ (93\%).

\subsection{Anionic Polymerization of Methyl Methacrylate}

The procedure similar to that mentioned above was utilized. Under nitrogen atmosphere $30 \mathrm{~mL}$ of dry THF was added to the flask. After cooling to $-78^{\circ} \mathrm{C}, 1 \mathrm{~mL}$ of sec-BuLi (1.00 mmol), and $10.6 \mathrm{~mL}$ of MMA (0.100 mol) were successively injected to the flask. After $1 \mathrm{~h}$, the content of flask was poured into methanol. Yield: $7.36 \mathrm{~g}$ $(73.6 \%)$.

\subsection{Synthesis of Polystyrene-Block-Poly (methyl methacrylate)}

Under nitrogen atmosphere $60 \mathrm{~mL}$ of dry THF was added to the flask. After cooling to $-78^{\circ} \mathrm{C}, 1 \mathrm{~mL}$ of sec-BuLi 
$(1.00 \mathrm{mmol})$, and $11.5 \mathrm{~mL}$ of St $(0.100 \mathrm{~mol})$ were successively injected to the flask. After $1 \mathrm{~h}, 0.18 \mathrm{~mL}$ of DPE ( $1.00 \mathrm{mmol})$ was added dropwise. After stirring for $30 \mathrm{~min}, 10.6 \mathrm{~mL}$ of MMA $(0.100 \mathrm{~mol})$ was injected to the mixture. After $3 \mathrm{~h}$, the reaction mixture was poured into methanol to precipitate the product. Yield: $18.0 \mathrm{~g}$ $(88 \%)$.

\subsection{Preparation of Seed Particles}

Polymers (total: $1.5 \mathrm{~g}$ ) were dissolved in $15 \mathrm{~mL}$ of dichloromethane. The solution was dispersed in $150 \mathrm{~mL}$ of $\mathrm{H}_{2} \mathrm{O}$ containing $0.5 \mathrm{~g}$ of PVA with a homogenizer (X520, CAT Scientific, US) to obtain 5 - $15 \mu \mathrm{m}$ sized droplets. Dispersion was transferred to $300-\mathrm{mL}$ beaker equipped with a mechanical stirrer, and the stirring was continued for $24 \mathrm{~h}$ to evaporate the solvent. The resulting particles were washed with distilled water with a centrifugation process.

\subsection{Seed Polymerization of Styrene and Methyl Methacrylate}

The dispersion of $0.5 \mathrm{~g}$ of seed particle in $40 \mathrm{~mL}$ of $\mathrm{H}_{2} \mathrm{O}$ containing $0.24 \mathrm{~g}$ of PVA and $0.1 \mathrm{~g}$ of SDS $0.1 \mathrm{~g}$ was charged into the $100-\mathrm{mL}$ three-necked flask with a mechanical stirrer. At $80 \mathrm{rpm}$, the emulsion of typically $0.5 \mathrm{~g}$ of monomer (St or MMA) and $40 \mathrm{mg}$ of AIBN in $10 \mathrm{~mL}$ of $\mathrm{H}_{2} \mathrm{O}$ containing $0.06 \mathrm{~g}$ of PVA and $0.02 \mathrm{~g}$ of SDS $0.02 \mathrm{~g}$ was dropwise added to the flask. After $24 \mathrm{~h}$, the temperature increased to $70^{\circ} \mathrm{C}$ to initiate polymerization. After polymerization for $4 \mathrm{~h}$, the product was washed with water and methanol, successively.

\subsection{Characterization}

Resulting PSt, PMMA and PS- $b$-PMMA were characterized with ${ }^{1} \mathrm{H}-\mathrm{NMR}$ (ECX 300, JEOL). Molecular weight and polydispersity (PD) were estimated by gelpermeation chromatography (GPC) equipped with JASCO 880PUpump, a column packed with styrene-divinylbenzene gel beads [25], a JASCO UV-970 detector, and GPC. The surface feature of microspheres after evaporation of dichloromethane was characterized by scanning electron microscope (SEM) (JSM-6510, JEOL). Chloroform was used as an eluent, and the molecular weight was calibrated using polystyrene standards (SHODEX). The specimens for SEM observations were prepared by coating a thin gold film on the sample with an ion sputter coater (IB-3, Eiko Engineering). The morphologies inside the polymer particles were observed with transmission electron microscope (TEM) (JEM-2100, JEOL, Japan). The TEM specimens were prepared by cutting ultrathin films (ca. $70 \mathrm{~nm}$ in thickness) from particles embedded in epoxy resin (Epok 812) with an ultramicrotome (EM UC7, Leica) and setting them on the copper meshes. The PSt domain was stained by exposing the specimens to the vapor of an aqueous $\mathrm{RuO}_{4}$ solution $(0.5 \%)$ for $90 \mathrm{~min}$ in a sealed bottle at room temperature.

\section{Results and Discussion}

\subsection{Preparation of Seed Particles}

Table 1 shows number average molecular weights, $M_{\mathrm{n}}$ of PS, PMMA and PS- $b$-PMMA, obtained from anion polymerization. All the seed particles were fabricated using these three polymers. PVA concentration was adjusted to $0.6 \mathrm{wt} \%$ so that regular, spherical particles with a diameter of $3-10 \mu \mathrm{m}$ are obtained.

As shown in Figure 1, spherical and smooth particles were obtained both from PS/PMMA (PS/PMMA = 1:1) polymer blend (a), and block copolymer (b). PSt and PMMA homopolymers also afforded the smooth particles. As Ma et al. reported, a typical core-shell type morphology (PMMA; shell, PSt; core) is formed for PSt/PMMA blend when PVA was used as a stabilizer [22], and microphase separated structure for PSt- $b$-PMMA.

Microspheres based on the ternary blends consisting of PSt, PMMA, and PSt- $b$-PMMA were fabricated. As shown in Figure 2, particles with a boundary were obtained. The addition of $10 \%$ of PSt- $b$-PMMA afforded particles with a line-shaped recess near the center of particles (Figure 2(a)). By the increase of the content of PSt$b$-PMMA to $20 \%$, particles with one smooth side and another rough side were obtained (Figure 2(b)). In the case of $30 \%$ and $40 \%$, the portion of rough surface seemed to increase compared with $20 \%$ particles. Figure 3 shows TEM images of particles based on ternary blend with 10\% of block copolymer. As PSt is preferentially stained, black sites represent PSt rich domain. According to typical three types of images (Figures 3(a)-(c)), plausible morphology inside the particle is schematically represented in Figure 3(d). It is found that the observed morphology is complex and intermediate between Janus and core-shell types. 
Table 1. Molecular weights of PSt, PMMA, and PSt- $b$-PMMA.

\begin{tabular}{ccccc}
\hline Polymer & $\begin{array}{c}M_{\mathrm{n}}^{\text {PSt }} \\
\left(\mathrm{g} \cdot \mathrm{mol}^{-1}\right)^{\mathrm{a}}\end{array}$ & $\begin{array}{c}M_{\mathrm{n}}^{\text {PMMA }} \\
\left(\mathrm{g} \cdot \mathrm{mol}^{-1}\right)^{\mathrm{a}}\end{array}$ & $\mathrm{PDI}^{\mathrm{b}}$ & $f_{\mathrm{PSt}^{\mathrm{c}}}$ \\
\hline PSt & 10,000 & - & 1.15 & - \\
PMMA & - & 12,000 & 1.25 & - \\
PSt- $b$-PMMA & 13,000 & 12,000 & 1.24 & 0.5 \\
\hline
\end{tabular}

${ }^{a}$ Determined by GPC; ${ }^{b}$ Polydispersity index determinded by GPC; ${ }^{c}$ Volume fraction of PSt.
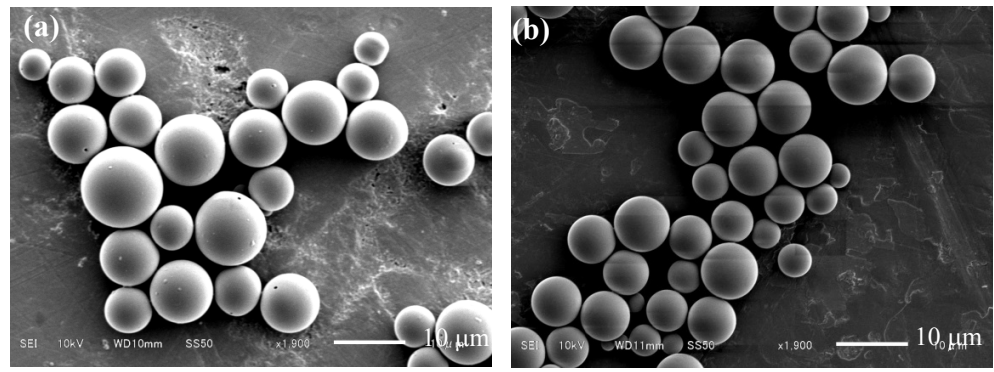

Figure 1. SEM photographs of microspheres based on PSt/PMMA (1:1) blend (a) and PSt- $b$-PMMA (b) particles.
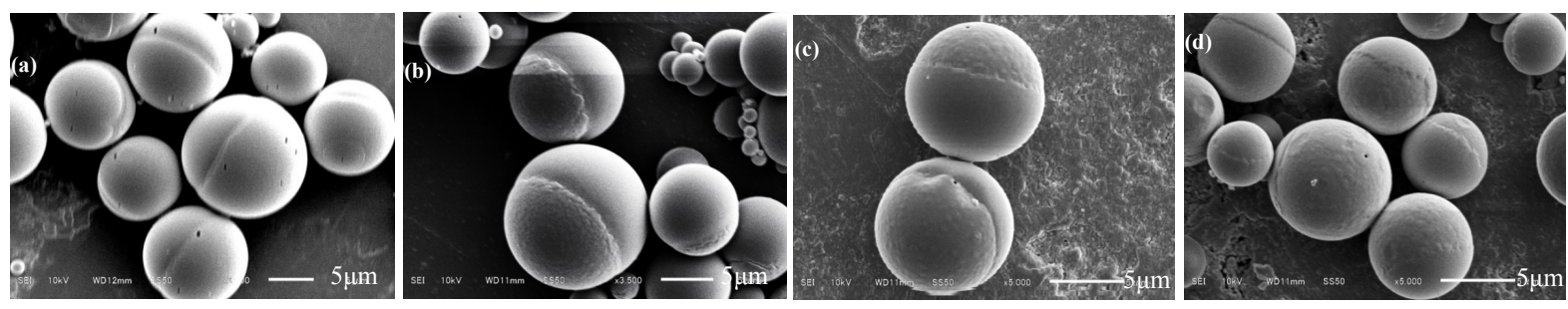

Figure 2. SEM photographs of microspheres based on PSt/PSt- $b$-PMMA/PMMA ternary blend. (a) $0.45 / 0.1 / 0.45$; (b) $0.4 / 0.2 / 0.4$; (c) $0.35 / 0.3 / 0.35$; (d) $0.3 / 0.4 / 0.3$.
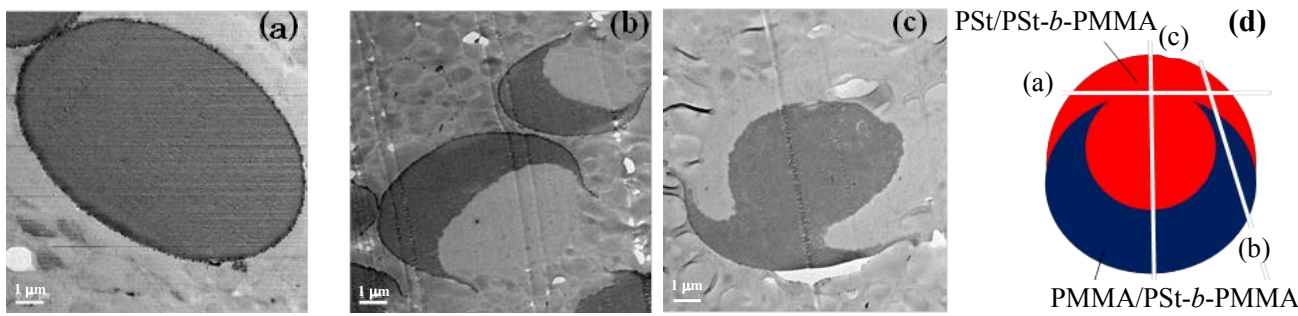

Figure 3. TEM photographs of ultrathin cross sections of $\mathrm{RuO}_{4}$-stained PSt/PSt- $b$-PMMA/PMMA composite particles prepared by solvent evaporation method (a)-(c). PSt/PSt- $b$-PMMA/PMMA $=0.45 / 0.1 / 0.45$. Illustration (d) schematically indicates the plausible phase structure and the directions of slices.

Taking the low content and the comparable segment molecular masses of the block copolymer into consideration, macroscopic phase separation is expected. In general, it is supposed that A-B type block or graft copolymer acts as a compatibilizer for the homopolymers (A and B) blend. Block or graft copolymer decreases the free energy of interface between the polymer blend to facilitate the mixing of two phases. In this report, it is reasonable that PSt- $b$-PMMA exists both in PSt-rich and PMMA-rich phases, which decreases the difference of the surface energies at the interface between both oil and water phases (PSt-rich phase and PMMA-rich phase). As a result, a part of PSt-rich phase can locate at the interface with water to exhibit the intermediate morphology. In this morphology, PMMA unit of PSt- $b$-PMMA in PSt-rich domain is considered to be located at the interface between polymer and water phases to decrease the interfacial energy. Therefore, surface roughness observed in Figures 2(b)-(d) was probably derived from the aggregation of PSt-b-PMMA near the surface of PSt-rich domain. 


\subsection{Seed Polymerization}

In a previous session, microspheres with various morphologies were obtained. Seed polymerizations of St and MMA were carried out in order to elucidate the effect of polymerization conditions on the final morphology.

At first, PSt/PMMA blend particles were utilized as seeds. Figure 4 shows SEM images of polymer particles after seed polymerization of MMA. When the amount of MMA was 10 or $100 \mathrm{wt} \%$ (a and b, respectively), no significant change was observed, and spherical particles with smooth surfaces were obtained. On the other hand, seed polymerization of $200 \mathrm{wt} \%$ of MMA afforded non-spherical particles with a bulge. From the optical microscope observation, particles swollen with MMA monomer show spherical shapes. Therefore it is considered that polymerization induced the irregular shape in the case of $200 \mathrm{wt} \%$ of MMA. As described in a previous session, seed particles have a core-shell morphology, hydrophilic PMMA shell, and hydrophobic PSt core. MMA monomer absorbed into seed particles is considered to be located in the shell part, and to be polymerized to form PMMA skin layer when the amount of MMA is not so high. With the increase of MMA monomer, the location of absorbed MMA extends over PSt core as well as PMMA shell. Polymerization of MMA in PSt phase induces the phase separation, resulting in the bulge.

Figure 5 shows SEM images of polymer particles after seed polymerization of St using PS/PMMA polymer blend seed particles. When 10 and $20 \mathrm{wt} \%$ of St was used, snowman-like particles were obtained (Figure 5(a) and Figure 5(b)). In the case of St, absorption of monomer also preferentially occurs at the PMMA shell, and stronger affinity between monomer and PSt core makes it possible to swell PSt core, resulting in protrusion of PSt/St mixture from the surface consisting of PMMA and St monomer. Indeed, Figure 6 indicated that snowman-like droplets were formed before polymerization both for 10 and $100 \mathrm{wt} \%$ of St. When 10 and $20 \mathrm{wt} \%$ of St was used, the viscosity in the protruded mixture is much higher than that for $100 \mathrm{wt} \%$ of St. Higher viscosity leads to fixation of the morphologies before polymerization. On the other hand, the decrease of viscosity in 100 $\mathrm{wt} \%$ mixture changes the shape from the swollen stage during polymerization (Figure 5(c)).
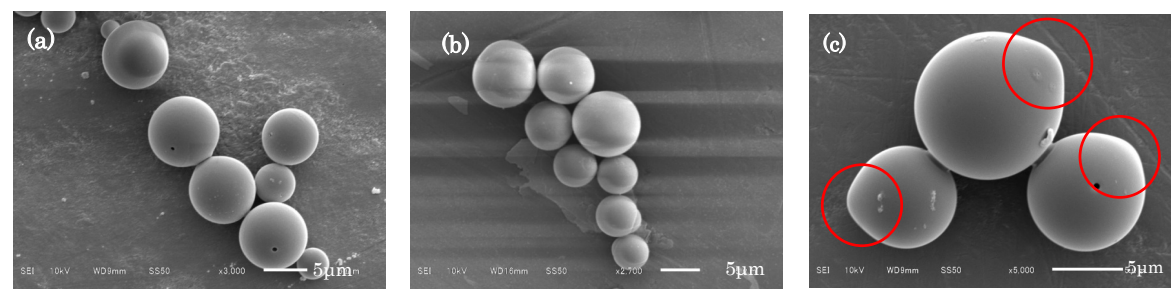

Figure 4. SEM photographs of polymer particles after seed polymerization of MMA using PSt/PMMA seed particles. (a) MMA $10 \mathrm{wt} \%$; (b) MMA $100 \mathrm{wt} \%$; (c) MMA $200 \mathrm{wt} \%$.
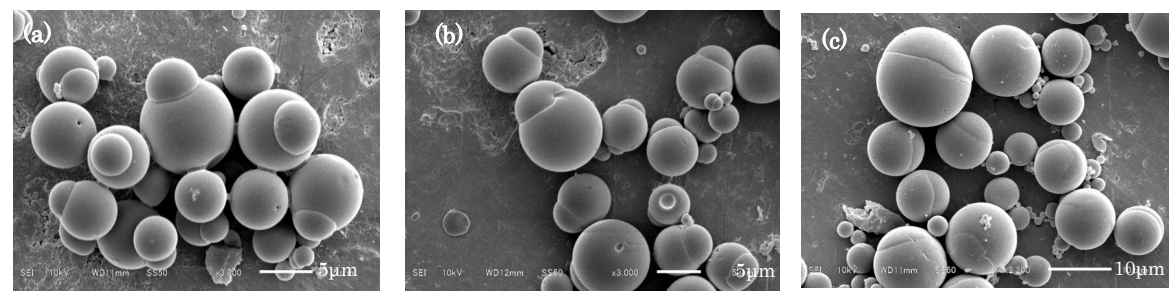

Figure 5. SEM photographs of polymer particles after seed polymerization of St using PSt/PMMA seed particles. (a) St 10 wt \%; (b) St $20 \mathrm{wt} \%$; (c) St $100 \mathrm{wt} \%$.
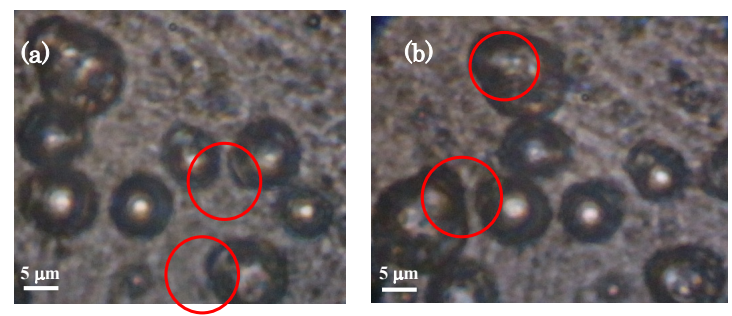

Figure 6. Optical microscope images of PS/PMMA seed particles after swelling with St. (a) St 10 wt $\%$; (b) St 100 wt $\%$. 
Next, PSt- $b$-PMMA microspheres were used as seed particles, and final morphologies were observed after seed polymerization of MMA and St monomers. Figure 7 shows SEM images of polymer particles after seed polymerization of MMA. Although the surface of seed particle is smooth (see Figure 1(b)), small protrusions with $100 \mathrm{~nm}$ size emerged after polymerization. At the stage of monomer absorption, surface is considered to consist of the mixture of MMA and the block copolymer. During polymerization, phase separation of resulting PMMA occurs near the surface to form the protrusions. With the increase of MMA amount, the surface tended to be covered with resulting PMMA more smoothly, and the surface roughness became discreet. Meanwhile, no significant morphology change was observed when St was used as a monomer as shown in Figure 8. Because of hydrophobicity of St, the absorbed monomer diffused toward the core direction compared with MMA. Polymerization of St and phase separation at the inside seems to have small effect on the surface morphology.

Finally seed polymerizations were carried out using ternary blend, and morphology changes were investigated. As discussed above, ternary blends consisting of PSt, PSt- $b$-PMMA, and PMMA show the unique bicompartment morphologies. Figure 9 shows SEM images of polymer particles after seed polymerization of $10 \mathrm{wt} \%$ of MMA. Seed particles with 20 or $30 \mathrm{wt} \%$ of the block copolymer have rough surface of one side due to the aggregation of PSt- $b$-PMMA near the surface of PSt-rich domain. As shown in Figure 9, the surface became smoother after polymerization. This is probably due to the averaging effect by the formation of skin layer of hydrophilic PMMA which is caused by the homogeneous distribution of MMA monomer on both surfaces. On the other hand, in the case of St (Figure 10), the surface of PMMA-rich phase became rougher with the increase of the block copolymer content (Figure 10(b) and Figure 10(c)). More St monomer is probably absorbed in PMMA-rich phase for the particles with the high content of PSt- $b$-PMMA, and polymerization induced phase separation inside the particle formed the protrusions.
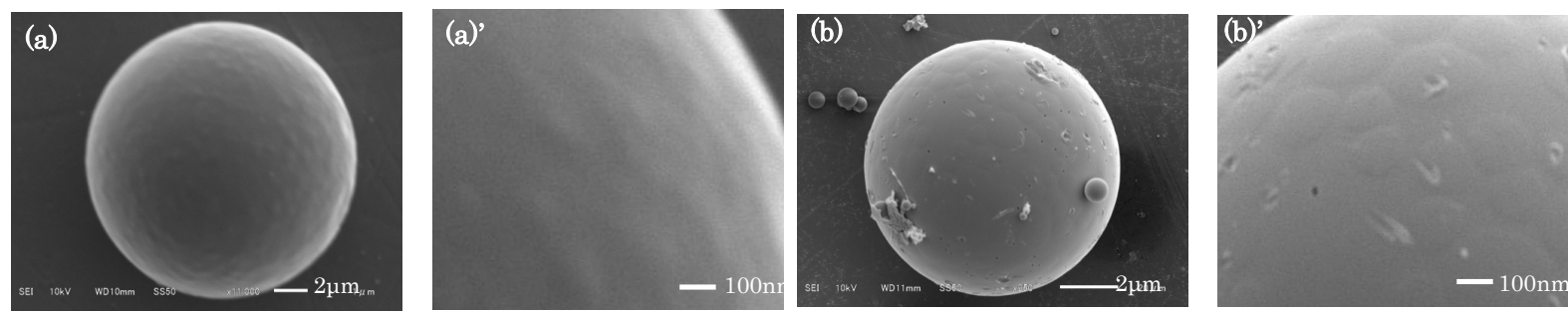

Figure 7. SEM photographs of polymer particles after seed polymerization of MMA using PSt- $b$-PMMA seed particles. (a) (a)' MMA $10 \mathrm{wt} \%$; (b) (b)' MMA $20 \mathrm{wt} \%$.
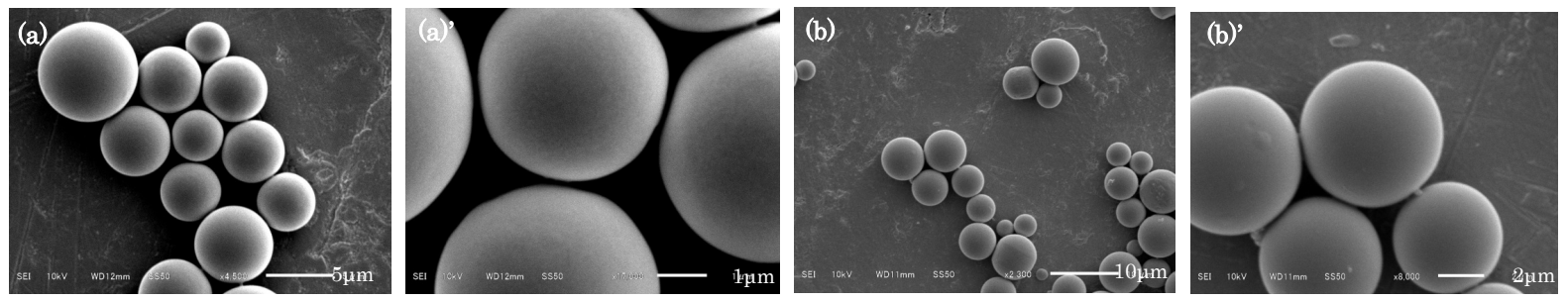

Figure 8. SEM photographs of polymer particles after seed polymerization of St using PS- $b$-PMMA seed particles. (a) St $10 \mathrm{wt} \%$; (b) St; $50 \mathrm{wt} \%$.
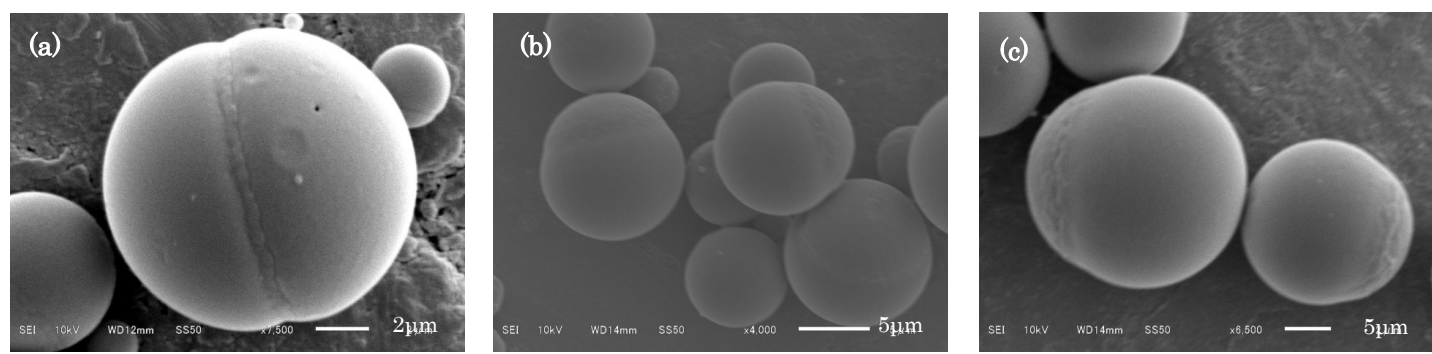

Figure 9. SEM photographs of polymer particles after seed polymerization of $10 \mathrm{wt} \%$ of MMA using PSt/PSt- $b$-PMMA/ PMMA composite seed particles. PSt/PSt- $b$-PMMA/PMMA: (a) $0.45 / 0.1 / 0.45$; (b) $0.4 / 0.2 / 0.4$; (c) $0.35 / 0.3 / 0.35$. 

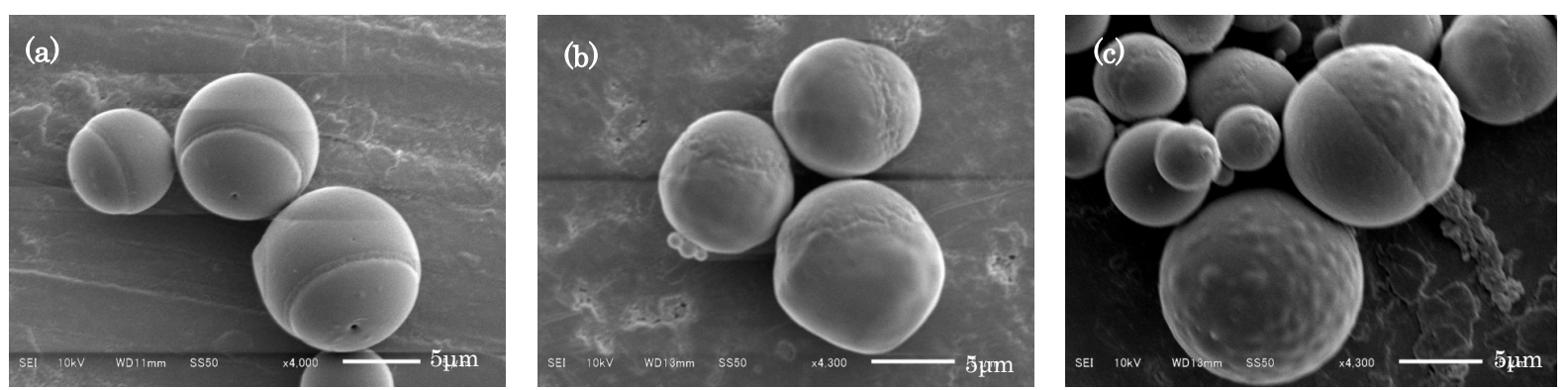

Figure 10. SEM photographs of polymer particles after seed polymerization of $10 \mathrm{wt} \%$ of St using PSt/PSt- $b$-PMMA/ PMMA composite seed particles. PSt/PSt- $b$-PMMA/PMMA: (a) $0.45 / 0.1 / 0.45$; (b) $0.4 / 0.2 / 0.4$; (c) $0.35 / 0.3 / 0.35$.

\section{Conclusion}

The microspheres with various types of surface and inside morphologies consisting of PSt/PMMA polymer blend, PSt- $b$-PMMA block copolymer and ternary (PSt/PSt- $b$-PMMA/PMMA) polymer blend were fabricated by a solvent evaporation method. Ternary blends afforded a unique morphology, intermediate between Janus and core-shell types. Bicompartment morphology was confirmed by TEM observation. The difference of interfacial tensions of two polymers with aqueous phase decreased by the addition of the block copolymer. Seed polymerizations of St and MMA were also conducted, and the morphologies change was investigated. The particles with snowman-like morphology were obtained by seed polymerization of St using PSt/PMMA binary blend microspheres as seed particles. Some seed polymerizations also controlled the surface roughness, i.e. polymerization of MMA in the block copolymer seed, and that of St in the ternary blend seed. The microspheres with various surfaces and inside structure have potential applications in photonic and electronic fields.

\section{References}

[1] Pancholi, K., Ahras N., Stride E. and Edirisinghe, M. (2009) Novel Electrohydrodynamic Preparation of Porous Chitosan Particles for Drug Delivery. Journal of Material Science: Material in Medicine, 20, 917-923. http://dx.doi.org/10.1007/s10856-008-3638-4

[2] Xie, J., Huang, J., Li, X., Sun, S. and Chen, X. (2009) Iron Oxide Nanoparticle Platform for Biomedical Applications. Current Medical Chemistry, 16, 1278-1294. http://dx.doi.org/10.2174/092986709787846604

[3] Perrier-Cornet, R., Heroguez, V., Thienpont, A., Babot, O. and Toupance, T. (2008) Functional Crosslinked Polymer Particles Synthesized by Precipitation Polymerization for Liquid Chromatography. Journal of Chromatography A, 1179, 2-8. http://dx.doi.org/10.1016/j.chroma.2007.09.064

[4] Garnett, M.C., Ferruti, P., Ranucci, E., Suardi, M.A., Heyde, M. and Sleat, R. (2009) Sterically Stabilized Self-Assembling Reversibly Cross-Linked Polyelectrolyte Complexes with Nucleic Acids for Environmental and Medical Applications. Biochemical Society Transactions, 37, 713-716. http://dx.doi.org/10.1042/BST0370713

[5] He, Y., Daniel, E.S., Klein, A. and El-Aasser, M.S. (1997) Grafting Behavior of n-Butyl Acrylate onto Poly(butadieneco-styrene) Latexes. Journal of Applied Polymer Science, 65, 511-523.

[6] Joensson, J.E., Hassander, H. and Toernell, B. (1994) Polymerization Conditions and the Development of a Core-Shell Morphology in PMMA/PS Latex Particles. 1. Influence of Initiator Properties and Mode of Monomer Addition. Macromolecules, 27, 1932-1937. http://dx.doi.org/10.1021/ma00085a042

[7] Cunningham, M.F., Mahabadi, H.K. and Wright, H.M. (2000) Supermicron Polymer Particles with Core-Shell Type Morphologies. Journal of Polymer Science, Part A: Polymer Chemistry, 38, 345-351.

[8] Oi, D.M., Bao, Y.Z., Huang, Z.M. and Weng, Z.X. (2006) Synthesis and Characterization of Poly(butyl acrylate)/Silica and Poly(butyl acrylate)/Silica/Poly(methyl methacrylate) Composite Particles. Journal of Applied Polymer Science, 99, 3425-3432. http://dx.doi.org/10.1002/app.22968

[9] Muroi, S., Hashimoto, H. and Hosoi, K. (1984) Morphology of Core-Shell Latex Particles. Journal of Polymer Science: Polymer Chemistry Edition, 22, 1365-1372. http://dx.doi.org/10.1002/pol.1984.170220617

[10] Daniel, E.S., Dimonie, V.L., El-Aasser, M.S. and Vanderhoff, J.W. (1990) Preparation of ABS (Acrylonitrile/Butadiene/Styrene) Latexes Using Hydroperoxide Redox Initiators. Journal of Applied Polymer Science, 41, 2463-2477. http://dx.doi.org/10.1002/app.1990.070410945

[11] Zhao, J., Yuan, H. and Pan, Z. (1994) Grafting Mechanism in SBR-St-MMA Core-Shell Emulsion Copolymerization. 
Journal of Applied Polymer Science, 53, 1447-1452. http://dx.doi.org/10.1002/app.1994.070531106

[12] El-Aasser, M.S., Hu, R., Dimonie, V.L. and Sperling, L.H. (1999) Morphology, Design and Characterization of IPNContaining Structured Latex Particles for Damping Applications. Colloids and Surfaces A: Physicochemical and Engineering Aspects, 153, 241-253. http://dx.doi.org/10.1016/S0927-7757(98)00448-8

[13] Yabu, H., Tajima, A., Higuchi, T. and Shimomura, M. (2007) Preparation of Polymer Nanoparticles by Self-Organization. Hyomen Kagaku, 28, 277-282. http://dx.doi.org/10.1380/jsssj.28.277

[14] Han, S.H., Ma, G.H., Du, Y.Z., Omi, S. and Gu, L.X. (2003) Preparation of Hemispherical Poly(4-vinylpyridine-cobutyl acrylate)/Poly(styrene-co-butyl acrylate) Composite Microspheres by Seeded Preswelling Emulsion Polymerization. Journal of Applied Polymer Science, 90, 3811-3821. http://dx.doi.org/10.1002/app.13094

[15] Hu, G., Yu, D., Zhang, J., Liang, H. and Cao, Z. (2011) Synthesis of Micron-Sized Poly(styrene-co-divinylbenzene) Hollow Particles from Seeded Emulsion by Using Swelling Solvents. Colloid Journal, 73, 557-564. http://dx.doi.org/10.1134/S1061933X11040077

[16] Zhang, Q., Yang, Z., Zhan, X. and Chen, F. (2009) Preparation and Structure Control of Hollow Polymer Particles: Influence of Seeded Emulsion Polymerization and Alkalization Treatment Process. Journal of Applied Polymer Science, 113, 207-215. http://dx.doi.org/10.1002/app.29631

[17] Okubo, M., Fujibayashi, T., Yamada, M. and Minami, H. (2005) Micron-Sized, Monodisperse, Snowman/ConfettiShaped Polymer Particles by Seeded Dispersion Polymerization. Colloid and Polymer Science, 283, 1041-1045. http://dx.doi.org/10.1007/s00396-004-1240-y

[18] Fujibayashi, T., Tanaka, T., Minami, H. and Okubo, M. (2010) Thermodynamic and Kinetic Consideration on the Morphological Stability of "Hamburger-Like" Composite Polymer Particles Prepared by Seeded Dispersion Polymerization. Colloid and Polymer Science, 288, 879-886. http://dx.doi.org/10.1007/s00396-010-2211-0

[19] Okubo, M., Fujibayashi, T. and Terada, A. (2005) Synthesis of Micron-Sized, Monodisperse Polymer Particles of Disk-Like and Polyhedral Shapes by Seeded Dispersion Polymerization. Colloid and Polymer Science, 283, 793-798. http://dx.doi.org/10.1007/s00396-004-1210-4

[20] Higuchi, T., Tajima, A., Motoyoshi, K., Yabu, H. and Shimomura, M. (2009) Suprapolymer Structures from Nanostructured Polymer Particles. Angewandte Chemie International Edition, 48, 5125-5128. http://dx.doi.org/10.1002/anie.200900002

[21] Higuchi, T., Tajima, A., Yabu, H. and Shimomura, M. (2008) Spontaneous Formation of Polymer Nanoparticles with Inner Micro-Phase Separation Structures. Soft Matter, 4, 1302-1305. http://dx.doi.org/10.1039/b800904j

[22] Ma, G.H., Nagai, M. and Omi, S. (1999) Study on Preparation and Morphology of Uniform Artificial PolystyrenePoly(methyl methacrylate) Composite Microspheres by Employing the SPG (Shirasu Porous Glass) Membrane Emulsification Technique. Journal of Colloid and Interface Science, 214, 264-282. http://dx.doi.org/10.1006/jcis.1999.6188

[23] Okubo, M., Saito, N. and Fujibayashi, T. (2005) Preparation of Polystyrene/Poly(methyl methacrylate) Composite Particles Having a Dent. Colloid and Polymer Science, 283, 691-698. http://dx.doi.org/10.1007/s00396-004-1209-x

[24] Saito, N., Kagari, Y. and Okubo, M. (2006) Effect of Colloidal Stabilizer on the Shape of Polystyrene/Poly(methyl methacrylate) Composite Particles Prepared in Aqueous Medium by the Solvent Evaporation Method. Langmuir, 22, 9397-9402. http://dx.doi.org/10.1021/la061298v

[25] Ogino, K., Sato, H., Tsuchiya, K., Suzuki, H. and Moriguchi, S. (1995) Synthesis of Monodisperse Macroreticular Styrene-Divinylbenzene Gel Particles by a Single-Step Swelling and Polymerization Method. Journal of Chromatography A, 699, 59-66. http://dx.doi.org/10.1016/0021-9673(95)00031-H 